\title{
Higher-accuracy van der Waals density functional
}

\author{
Kyuho Lee, ${ }^{1}$ Éamonn D. Murray, ${ }_{1}^{1}$ Lingzhu Kong, ${ }^{1}$ Bengt I. Lundqvist, ${ }^{2,3}$ and David C. Langreth ${ }^{1}$ \\ ${ }^{1}$ Department of Physics and Astronomy, Rutgers University, Piscataway, New Jersey 08854-8019, USA \\ ${ }^{2}$ Department of Applied Physics, Chalmers University of Technology, SE-41296 Göteborg, Sweden \\ ${ }^{3}$ Center for Atomic-scale Materials Design, Department of Physics, Technical University of Denmark, \\ DK-2800 Kongens Lyngby, Denmark
}

(Received 12 March 2010; revised manuscript received 8 July 2010; published 4 August 2010)

\begin{abstract}
We propose a second version of the van der Waals density functional of Dion et al. [Phys. Rev. Lett. 92, 246401 (2004)], employing a more accurate semilocal exchange functional and the use of a large- $N$ asymptote gradient correction in determining the vdW kernel. The predicted binding energy, equilibrium separation, and potential-energy curve shape are close to those of accurate quantum chemical calculations on 22 duplexes. We anticipate the enabling of chemically accurate calculations in sparse materials of importance for condensed matter, surface, chemical, and biological physics.
\end{abstract}

DOI: 10.1103/PhysRevB.82.081101

PACS number(s): 71.15.Mb, 31.15.E-

The van der Waals (vdW) attraction is a quantummechanical phenomenon with charge fluctuations in one part of an atomic system that are electrodynamically correlated with charge fluctuations in another. The vdW force at one point thus depends on charge events at another region and is a truly nonlocal correlation effect.

Methods for accurately calculating the vdW interactions are critical to understanding sparse matter, including bulk solids (e.g., layered materials, molecular crystals, and polymers), surface phenomena (e.g., adsorption, water overlayers, and gas separation and storage), and biostructures (e.g., DNA and protein structure).

The exact density functional contains the vdW forces. Unfortunately, we do not have access to it but approximate versions are abundant. Commonly, the local-density approximation (LDA) and generalized gradient approximation are used with quite some success for dense matter, including hard materials and covalently bound molecules. They depend on the density in local and semilocal ways, respectively, however, and give no account of the fully nonlocal vdW interaction.

First-principles approaches for how vdW can be treated in DFT were first proposed for the asymptotic interaction between fragments. ${ }^{1-3}$ These ultimately evolved into the van der Waals density functional (vdW-DF) for arbitrary geometries. ${ }^{4-6}$ Despite its success for describing dispersion in a breadth of systems better than any other nonempirical method, ${ }^{7}$ vdW-DF overestimates equilibrium separations , $5,7-12$ and underestimates hydrogen-bond strength. ${ }^{13,14}$

In this Rapid Communication, we propose a second version of the van der Waals density functional (vdW-DF2) employing a more accurate semilocal exchange functional PW86 (Refs. 15 and 16) and the use of a large- $N$ asymptote gradient correction ${ }^{17}$ in determining the vdW kernel. By making a full comparison of potential-energy curves (PECs) with accurate quantum chemistry (QC) results for 22 molecular duplexes, we show that vdW-DF2 substantially improves (i) equilibrium separations, (ii) hydrogen-bond strengths, and (iii) vdW attractions at intermediate separations longer than the equilibrium ones. The improvement in (iii), found via a full PEC comparison, is most critical for important "real-life" applications to sparse matter and biological matter where it is impossible for basic structural units to assume the same separations they would have as binary units in vacuo.

First, we replace Perdew-Burke-Ernzerhof (revPBE) exchange functional ${ }^{18}$ with $\mathrm{PW} 86,{ }^{15,16}$ because revPBE is generally too repulsive near the equilibrium separation, ${ }^{8}$ and can bind spuriously by exchange alone, although less so than most other local or semilocal functionals. Hence, other exchange functionals ${ }^{19,20}$ have been proposed. Recent performance studies of various exchange functionals for weakly interacting atoms ${ }^{21}$ and molecules, ${ }^{22}$ however, show PW86, with an enhancement factor proportional to $s^{2 / 5}$ at large reduced density gradient $s$, to give the most consistent agreement with Hartree-Fock (HF) results, without spurious exchange binding. It also is a good match ${ }^{23}$ for the vdW-DF2 correlation kernel, introduced below, although no others were tried.

The key to the vdW-DF method is the inclusion of a longrange piece of the correlation energy, $E_{c}^{n l}[n]$, a fully nonlocal functional of the density $n$. This piece is evaluated using a "plasmon" pole approximation for the inverse dielectric function, which satisfies known conservation laws, limits, sum rules, and invariances. ${ }^{4} \mathrm{~A}$ single parameter model for the pole position was adopted, with the pole residue set by the law of charge-current continuity ( $f$-sum rule), and the pole position at large wave vector set by the constraint that there be no self-Coulomb interaction. The single parameter is determined locally from electron-gas energy input via gradient corrected LDA. ${ }^{4}$

The nonlocal piece of the correlation energy in both vdW-DF and vdW-DF2 is of the form

$$
E_{c}^{n l}[n]=\int d^{3} r \int d^{3} r^{\prime} n(\mathbf{r}) \phi\left(\mathbf{r}, \mathbf{r}^{\prime}\right) n\left(\mathbf{r}^{\prime}\right) .
$$

The kernel $\phi$ is given as a function of $R f(\mathbf{r})$ and $R f\left(\mathbf{r}^{\prime}\right)$, where $R=\left|\mathbf{r}-\mathbf{r}^{\prime}\right|$ and $f(\mathbf{r})$ is a function of $n(\mathbf{r})$ and its gradient. In fact $f(\mathbf{r})$ is proportional to the exchange-correlation energy density $\epsilon_{x c}$ of a gradient corrected LDA at the point $\mathbf{r}$. 

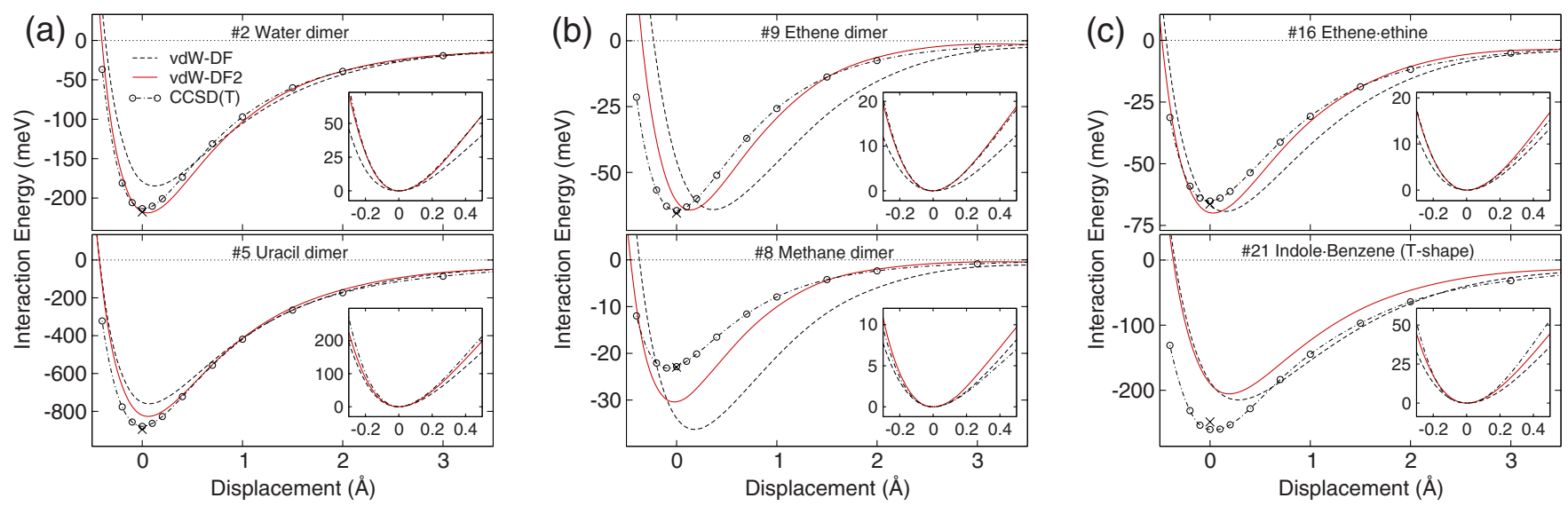

FIG. 1. (Color online) PECs for the best and the worst case of (a) hydrogen-bonded, (b) dispersion-dominated, and (c) mixed duplexes. $\operatorname{CCSD}(\mathrm{T})$ QC PECs (dashed-dotted lines with circles taken from Ref. 33) and the reference energies (cross marks taken from Ref. 32) at the geometry of Ref. 31 are also shown. The shapes near minima are compared in inset figures where PECs are aligned to have the common minimum point. For all the other S22 duplexes, see supplementary material.

This arose from the approximately implemented requirement that the dielectric function implied by the plasmon pole model should give an exchange-correlation energy semilocally consistent with a gradient corrected electron gas. We call the semilocal functional that fixes $f(\mathbf{r})$ in Eq. (1) the internal functional.

The above is easier to understand for two separate molecules, although the arguments apply equally well to a pair of high-density regions of a sparse material. The long-range $\mathrm{vdW}$ attraction implied by Eq. (1) occurs from the contribution where $\mathbf{r}$ is on one molecule and $\mathbf{r}^{\prime}$ on the other. The definition of $f(\mathbf{r})$ and $f\left(\mathbf{r}^{\prime}\right)$ varies continuously and independently at each point according to $\epsilon_{x c}(\mathbf{r})$ and $\epsilon_{x c}\left(\mathbf{r}^{\prime}\right)$. The quantity $\epsilon_{x c}$ is taken to consist of a gradient corrected LDA. In the first version of $\mathrm{vdW}-\mathrm{DF},{ }^{4}$ the gradient correction was obtained from a gradient expansion ${ }^{24}$ for the slowly varying electron gas. ${ }^{25,26}$ More appropriate is a functional that gives accurate energies for molecules, however. When $\mathbf{r}$ and $\mathbf{r}^{\prime}$ are each in a separate moleculelike region, with exponentially decaying tails between them, $f(\mathbf{r})$ and $f\left(\mathbf{r}^{\prime}\right)$ can both be large and give key contributions to a vdW attraction. For this case (including perhaps even a molecule near a surface) the large- $N$ asymptote ${ }^{27,28}$ and the exchange energy asymptotic series for neutral atoms provide a more accurate approximation. In fact, the exchange parameter ${ }^{26} \beta$ of the B88 exchange functional, ${ }^{29}$ successfully used in the vast majority of DFT calculations on molecules, can be derived from first principles using the large- $N$ asymptote ${ }^{17}$ as can the LDA exchange. It seems obvious, then, that vdW-DF results should be improved if the second-order expansion of the exchange in gradients is replaced by the second-order large- $N$ expansion. Interestingly, PW86R functional, selected as the overall exchange functional for different reasons, also follows the large- $N$ behavior for small reduced gradient $s$ values down to $\sim 0.1$, where it reverts to the form of slowly varying electron-gas limit.

Thus we use 2.222 times larger exchange gradient coefficient, a value based on agreement between derived ${ }^{17,27,28}$ and

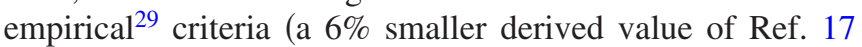
only gives a marginal improvement). Assuming that the screened exchange term ${ }^{30}$ increases in the same proportion as gradient exchange itself, finally we get the appropriate gradient coefficient in the " $Z$ " notation ${ }^{26}$ which is multiplied by 2.222. Summarizing: while $Z_{a b}=-0.8491$ in vdW-DF, $Z_{a b}=$ -1.887 in vdW-DF2, implying changes in the internal functional.

The performance of our energy functional is assessed via comparisons with the accurate $\mathrm{S} 22$ reference data set $\mathrm{t}^{31,32}$ and PECs (Ref. 33) based on QC calculations at the level of coupled-cluster with single and double and perturbative triple excitations $[\operatorname{CCSD}(\mathrm{T})]$ with extrapolation to the complete basis set limit. These 22 small molecular duplexes for the noncovalent interactions typical in biological molecules include hydrogen-bonded, dispersion-dominated, and mixed duplexes. Recent evaluation ${ }^{13}$ of the performance of the vdW-DF for S22 shows it to be quite good, except for H-bonded duplexes, where vdW-DF underestimates the binding energy by about $15 \%$.

Calculations are performed by a plane-wave code and an efficient vdW algorithm ${ }^{34}$ with Troullier-Martins-type normconserving pseudopotentials. Spot comparison with all electron calculations using large basis sets indicates a calculational accuracy of $\sim 1 \%$, actually better than that of most of PAW potentials supplied in various standard codes. Large box sizes were used to control spurious electrostatic interactions between replicas. See supplementary material ${ }^{35}$ for further details.

Figure 1 shows a typical PEC for each kind of interaction. To make a direct comparison to the $\mathrm{QC}$ results, the PECs are calculated at the same geometry of those of the $\operatorname{CCSD}(\mathrm{T})$ PEC calculations ${ }^{33}$ (shown as dashed-dotted lines with circles), where each molecule is kept in its S22 geometry ${ }^{31}$ and moved along the line defined by the center-of-mass coordinates of two molecules without any rotation. Overall, the vdW-DF2 PECs (solid lines) show a remarkable agreement with QC ones for all separations and for all three types of interactions. The shapes of the PECs near the minima, important for vibrational frequencies, are greatly improved (see inset figures where PECs are aligned to have the common minimum point). More importantly the strength of vdW at- 


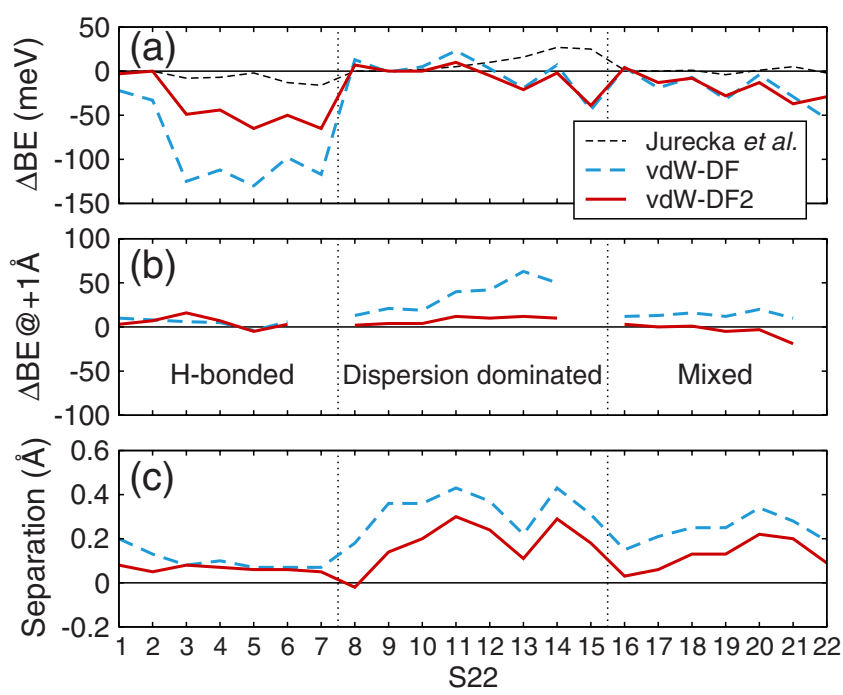

FIG. 2. (Color online) Comparison for the S22 duplexes of (a) binding energy predicted by Jurecka et al. (Ref. 31), vdW-DF (Ref. 4), and the present work (vdW-DF2). (b) Binding energy at a separation $1 \AA$ larger than the equilibrium one and (c) equilibrium separations. The ordinates give the respective deviations of these quantities from the reference values taken from Takatani et al. (Ref. 32) for panel (a), Molnar et al. (Ref. 33) for panel (b), and Jurecka et al. (Ref. 31) for panel (c).

traction at larger distances is weakened in agreement with QC, especially for vdW-bonded duplexes [Fig. 1(b)]. In other words, the original vdW-DF overestimates vdW attraction at intermediate separations beyond the equilibrium separation, although its minimum energy is accurate. This has special importance in sparse condensed matter. An example will be given below.

The PECs tend to turn up slightly earlier when approaching the repulsion regime at small separations. This quite universal feature might be due to PW86 exchange functional which is slightly more repulsive than HF at short distances. ${ }^{22}$ For duplexes whose large distance asymptote is dominated by dispersion (here methane dimer, ethene dimer, and benzene-methane), vdW-DF2 will have weaker attraction in the asymptotic region and smaller $C_{6}$ coefficients than vdWDF, which (at least for the methane dimer) already gives a $C_{6}$ coefficient close to experiment. ${ }^{6}$ Recently, this prediction was verified in detail for various duplexes. ${ }^{36}$ However, in the region of our calculations, such deterioration is not pervasive. In any case, neither the vdW-DF curves nor the QC curve have reached their asymptotic limit for any of the above three cases. The remainder of the 22 duplexes have asymptotic forms dominated by electrostatics.

In Fig. 2, we summarize the calculated binding energies at equilibrium separation, at equilibrium separation plus $1 \AA$, and the equilibrium separations themselves, with each quantity given as a deviation from that implied by the reference calculations. $^{31-33}$ Each subfigure clearly shows one of the three major improvements. (i) Hydrogen-bond strengths. The mean absolute deviation (MAD) of binding energy for hydrogen-bonded duplexes is decreased from 91 to $40 \mathrm{meV}$. (ii) Interaction at intermediate separations. The MAD of binding energy at a separation $1 \AA$ larger than the equilib- rium one is reduced from 35 to $8 \mathrm{meV}$ for the dispersiondominated group and also substantially for the mixed group. (iii) Equilibrium separations. MAD is reduced from 0.23 to $0.13 \AA$. Overall, the vdW-DF2 binding energies are within $50 \mathrm{meV}$ of the reference except for duplexes 5 and 7 . The MAD of binding energy is decreased from 41 to $22 \mathrm{meV}$ (13\% to $7.6 \%)$. As a final tidbit, we note that in vdW-DF2 the MAD for the equilibrium energy of the dispersiondominated complexes has been reduced to $11 \mathrm{meV}$, which is equal to the MAD of the work of Jurecka et al. ${ }^{31}$ which until very recently ${ }^{32}$ was considered the "gold standard" for accuracy in quantum chemical calculations on this group.

The higher accuracy of vdW-DF2 persists in the extended systems we tested. As such applications, we calculated: (i) the graphite interlayer binding energy and spacing. The binding energy is on a par with that of vdW-DF which is already good. Interlayer spacing is about $2 \%$ shorter than that by vdW-DF, in better agreement with experiment. (ii) $\mathrm{H}_{2}$ adsorption within two different metal-organic frameworks (MOFs), $\mathrm{Zn}_{2}$ (BDC) $)_{2}$ (TED) and MOF-74. In the former, the binding at the highest binding site $(57 \mathrm{meV}$ including zero point) is in good agreement with heat of adsorption measurement $(52 \mathrm{meV})$, whereas vdW-DF overestimates it by $60 \%$. This demonstrates the importance of accurate intermediaterange interaction. For comparison, the binding energy of hydrogen to benzene, one of building blocks of $\mathrm{Zn}_{2}$ (BDC) $)_{2}$ (TED), is almost the same for both functionals at the equilibrium separation. At larger separations, however, vdW-DF substantially overestimates the binding. In case of MOF-74, $\mathrm{H}_{2}$ positions are experimentally known. VdW-DF2 shows its higher accuracy in predicting separations between hydrogens and nearby atoms (improved by $0.1-0.2 \AA$ ). The strongest binding comes from an unsaturated metal atom, rather than more distant structures, and the binding energy, already accurate in vdW-DF, is little changed in vdW-DF2. We also tested vdW-DF2 on the internal structure of a water molecule, as an example of strong chemical bonds in molecules. We find that vdW-DF2 is on par with PBE. For details of all tests, see supplementary material.

In summary, we have presented an enhanced version of vdW-DF, denoted by vdW-DF2, which can be implemented via simple modifications of existing codes. It results in significant improvements in equilibrium spacings between noncovalently bound complexes, as well as in binding energy, especially when hydrogen bonding plays a role. We make a full comparison of PECs of both functionals with accurately known results for a set of 22 complexes and also apply it to extended solid systems, finding favorable results for the new functional. Thus, we expect our method to have important applications in a wide range of fields, including condensed matter and materials physics, chemical physics, and the physics of biological materials.

We thank V. Cooper, A. Gulans, and R. Nieminen for discussions, P. Elliott and K. Burke for an early copy of Ref. 17 , and D. Case for the use of his computer cluster for several spot checks. Work principally supported by NSF under Grant No. DMR-0801343; work on MOFs by DOE under Grant No. DE-FG02-08ER46491; D.C.L. work at KITP by NSF under Grant No. PHY05-51164; and B.I.L. work by the Lundbeck Foundation via CAMD. 
${ }^{1}$ Y. Andersson, D. C. Langreth, and B. I. Lundqvist, Phys. Rev. Lett. 76, 102 (1996).

${ }^{2}$ J. F. Dobson and B. P. Dinte, Phys. Rev. Lett. 76, 1780 (1996).

${ }^{3}$ W. Kohn, Y. Meir, and D. E. Makarov, Phys. Rev. Lett. 80, 4153 (1998).

${ }^{4}$ M. Dion, H. Rydberg, E. Schröder, D. C. Langreth, and B. I. Lundqvist, Phys. Rev. Lett. 92, 246401 (2004).

${ }^{5}$ T. Thonhauser, V. R. Cooper, S. Li, A. Puzder, P. Hyldgaard, and D. C. Langreth, Phys. Rev. B 76, 125112 (2007).

${ }^{6} \mathrm{~A}$ recent variant denoted vdW-DF-09 by its authors, O. A. Vydrov and T. Van Voorhis, J. Chem. Phys. 130, 104105 (2009), introduces some empiricism but has been applied to too few systems to assess usefulness; a more recent functional, $\mathrm{O}$. A. Vydrov and T. Van Voorhis, Phys. Rev. Lett. 103, 063004 (2009), has been the subject of further discussion; D. C. Langreth and B. I. Lundqvist, ibid. 104, 099303 (2010); O. A. Vydrov and T. Van Voorhis, ibid. 104, 099304 (2010).

${ }^{7}$ D. C. Langreth et al., J. Phys.: Condens. Matter 21, 084203 (2009).

${ }^{8}$ A. Puzder, M. Dion, and D. C. Langreth, J. Chem. Phys. 124, 164105 (2006).

${ }^{9}$ S. D. Chakarova-Käck, E. Schröder, B. I. Lundqvist, and D. C. Langreth, Phys. Rev. Lett. 96, 146107 (2006).

${ }^{10}$ L. Kong, G. Román-Pérez, J. M. Soler, and D. C. Langreth, Phys. Rev. Lett. 103, 096103 (2009).

${ }^{11}$ K. Toyoda et al., Surf. Sci. 603, 2912 (2009).

${ }^{12}$ L. Romaner et al., New J. Phys. 11, 053010 (2009).

${ }^{13}$ A. Gulans, M. J. Puska, and R. M. Nieminen, Phys. Rev. B 79, 201105(R) (2009).

${ }^{14}$ A. K. Kelkkanen, B. I. Lundqvist, and J. K. Nørskov, J. Chem. Phys. 131, 046102 (2009).

${ }^{15}$ J. P. Perdew and Y. Wang, Phys. Rev. B 33, 8800(R) (1986).

${ }^{16}$ We recommend the refitted version PW86R (see Ref. 22, Table 2 ) for increased accuracy on the $\sim 1 \%$ level. It was used for the vdW-DF2 calculations here.

${ }^{17}$ P. Elliott and K. Burke, Can. J. Chem. 87, 1485 (2009).

${ }^{18}$ Y. Zhang and W. Yang, Phys. Rev. Lett. 80, 890 (1998).

${ }^{19}$ V. R. Cooper, Phys. Rev. B 81, 161104 (2010).

${ }^{20}$ J. Klimeš, D. R. Bowler, and A. Michaelides, J. Phys.: Condens. Matter 22, 022201 (2010).

${ }^{21}$ F. O. Kannemann and A. D. Becke, J. Chem. Theory Comput. 5, 719 (2009).

${ }^{22}$ E. D. Murray, K. Lee, and D. C. Langreth, J. Chem. Theory Comput. 5, 2754 (2009).

${ }^{23}$ VdW-DF with PW86 exchange corrects equilibrium separations but overestimates bindings and vdW attractions beyond equilib- rium separations which will be fixed by new vdW-DF2 correlation kernel. See Fig. 4 in supplementary material (Ref. 35).

${ }^{24}$ D. C. Langreth and S. H. Vosko, Adv. Quantum Chem. 21, 175 (1990).

${ }^{25}$ The second-order expansion of the vdW interaction, the "c" term in the notation of Ref. 24, was omitted so that only the "a" and "b" terms are retained, hence the notation $Z_{a b}$. See Appendix B of Ref. 5. for a full discussion.

${ }^{26}$ The relation between $Z$ and other notations for the gradient coefficient: $Z=-9 \mu=-48 \pi\left(3 \pi^{2}\right)^{1 / 3} \beta$. For pure exchange, $Z=$ $-10 / 9$ in the gradient expansion, while for B88, $Z=-2.469$.

${ }^{27}$ J. Schwinger, Phys. Rev. A 22, 1827 (1980).

${ }^{28}$ J. Schwinger, Phys. Rev. A 24, 2353 (1981).

${ }^{29}$ A. D. Becke, Phys. Rev. A 38, 3098 (1988).

${ }^{30}$ This screening term is considerably smaller than the gradient exchange term. We expect that as an estimate, we can without major overall error simply assume that it increases in the same proportion as gradient exchange itself. This ansatz, unlike other simple approaches we might have taken, also assures that the effect of the 10/7 factor enhancing Sham exchange continues to cancel out of exchange and correlation together as it should (Ref. 24).

${ }^{31}$ P. Jurečka, J. Šponer, J. Černý, and P. Hobza, Phys. Chem. Chem. Phys. 8, 1985 (2006).

${ }^{32}$ T. Takatani et al., J. Chem. Phys. 132, 144104 (2010).

${ }^{33}$ L. F. Molnar, X. He, B. Wang, and K. M. Merz, J. Chem. Phys. 131, 065102 (2009).

${ }^{34}$ G. Román-Pérez and J. M. Soler, Phys. Rev. Lett. 103, 096102 (2009); we adapted SIESTA, P. Ordejón, E. Artacho, and J. M. Soler, Phys. Rev. 53, 10441(R) (1996); J. M. Soler et al., J. Phys.: Condens. Matter 14, 2745 (2002); vdW code for use within a modified version of ABINIT, X. Gonze et al., Comput. Mater. Sci. 25, 478 (2002). The vdW interaction was treated fully self-consistently including forces. The computational costs are the same with vdW-DF. See supplementary material for more details (Ref. 35).

${ }^{35}$ See supplementary material at http://link.aps.org/supplemental/ 10.1103/PhysRevB.82.081101 for a table giving our calculated binding energies and equilibrium separations in vdW-DF and vdW-DF2 for all the S22 duplexes, as well as figures showing those S22 PECs omitted in this Rapid Communication.

${ }^{36}$ O. A. Vydrov and T. Van Voorhis, Phys. Rev. A 81, 062708 (2010). 